\title{
Robot Expressionism Through Cartooning
}

\author{
James E. Young \\ University of Calgary \\ 2500 University Drive \\ Calgary, AB, Canada \\ jyoung@cpsc.ucalgary.cs
}

\author{
Min Xin \\ University of Calgary \\ 2500 University Drive \\ Calgary, AB, Canada \\ xinm@cpsc.ucalgary.ca
}

\author{
Ehud Sharlin \\ University of Calgary \\ 2500 University Drive \\ Calgary, AB, Canada \\ ehud@cpsc.ucalgary.ca
}

\begin{abstract}
We present a new technique for human-robot interaction called robot expressionism through cartooning. We suggest that robots utilise cartoon-art techniques such as simplified and exaggerated facial expressions, stylised text, and icons for intuitive social interaction with humans. We discuss practical mixed reality solutions that allow robots to augment themselves or their surroundings with cartoon art content. Our effort is part of what we call robot expressionism, a conceptual approach to the design and analysis of robotic interfaces that focuses on providing intuitive insight into a robotic state as well as artistic quality of interaction. Our paper discusses a variety of ways that allow robots to express cartoon art, and details a test bed design, implementation, and preliminary evaluation. We describe our test bed, Jeeves, which uses a Roomba, an iRobot vacuum cleaner robot, and a mixed-reality system as a platform for rapid prototyping of cartoon-art interfaces. Finally, we present a set of interaction content scenarios which use the Jeeves prototype: trash roomba, the recycle police, and clean tracks, as well as initial user evaluation of our approach.
\end{abstract}

\section{Categories and Subject Descriptors}

H.5.2 [Information interfaces and presentation]: User Interfaces-Graphical user interfaces (GUI), Interaction styles, Theory and methods

\section{Keywords}

Mixed reality, Human-robot interaction

\section{INTRODUCTION}

When we communicate with other humans, through any medium such as speech, gestures, the written word or art, we rely on complex levels of inter-personal common ground and understanding as a base for communication. However, when we interact with robots, common ground is very limited, if it exists at all; robots think in the foreign language of bits and bytes, a language we humans cannot inherently understand. This poses a problem, as robot technology is quickly advancing towards autonomous and intelligent robots; robots which will soon enter our everyday environments. As this happens, it is important that we teach robots to communicate effectively using language and techniques understood by the general population.

There has been a recent shift in Human-Robot Interaction (HRI) toward the development of social robots which use human communication techniques to interact with people.

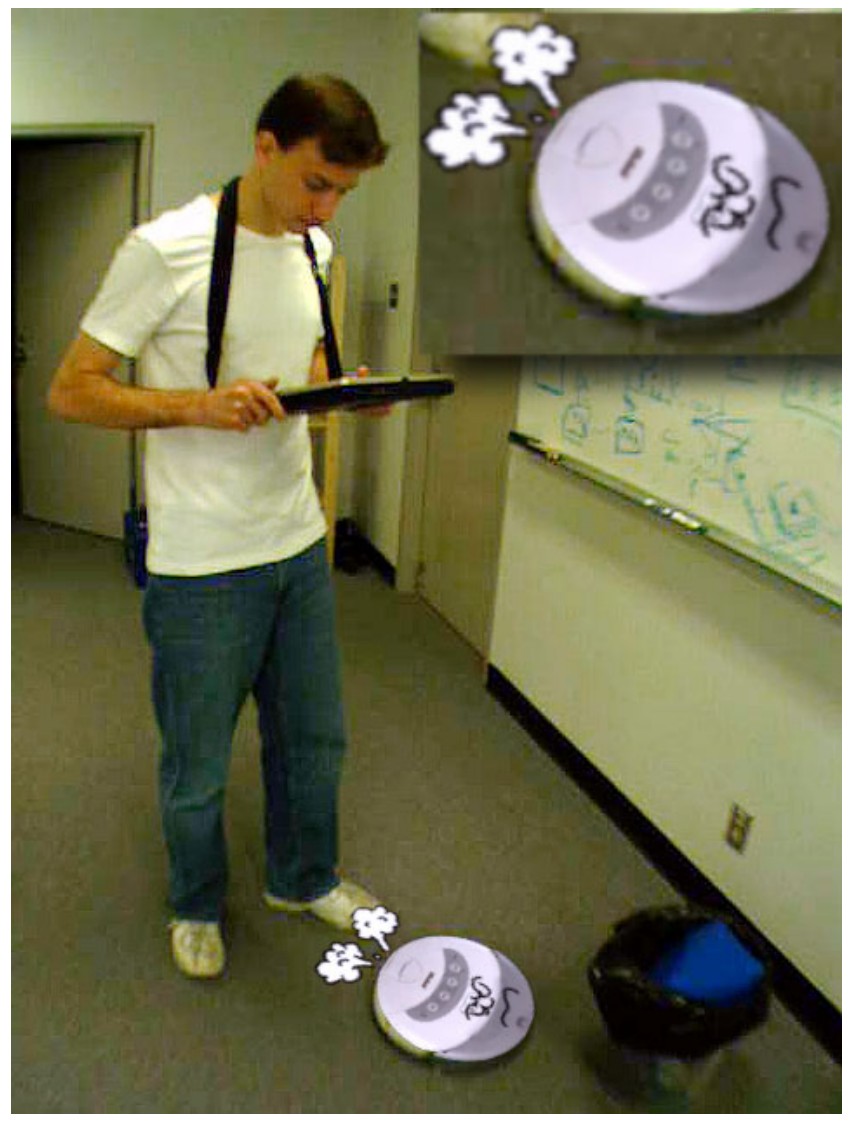

Figure 1: A Roomba using cartoon art to communicate with a user.

This approach is fuelled by an increase in robot autonomy and their entrance into the everyday lives of the general public. In favour of social robots, Human-Computer Interaction (HCI) guru Donald Norman argues that humans have the tendency to interpret the state of things by emoting and anthropomorphising, and that robots should take advantage of this tendency by displaying their state using human-like communication techniques, similar to how a person or a pet does [17].

In addition to more direct methods like gestures and speech, humans use artistic or symbolic methods such as the written 
word or drawings for communication. We believe that these methods can be used by robots for expression and communication. In particular, we believe that modern cartoon art, the simplified artistic and visual language found in comic books and animated cinema, can provide powerful expression mechanisms for robots.

Robots that use cartoon art (as shown in Figure 1) will have a rich communication vocabulary that is widely understood by the general public. These robots will be able to utilise meaningful cartoon-like annotations, many of which are able to express human-like emotion. For example, a robot may place sweat drops on its forehead to express fatigue and the need to recharge. Using such cartoon art to express a low battery state will enable robots to communicate clearly and intuitively across many language and cultural barriers, whereas the meaning of a "low-battery" light may not be clear or users may not take the time to read, interpret, and understand a battery level meter. A robot may also use simplified cartoon-like facial expressions to convey happiness for completing a mission or fear for not completing a task on time. From a human's point of view, these expressions foster an intuitive understanding of the robot's internal state, tasks, goals, and intentions, and can improve the effectiveness of social interaction in comparison to low-level and limited expressions such as blinking lights or text messages.

We implement the cartoon-art system using Mixed Reality (MR), a technology that utilises displays (such as a projector, Head-Mounted Display (HMD), or wall-display) to visually augment a user's vision with computer graphics; in this case, cartoon elements. MR allows for computer graphics to be placed directly within the physical environment, and robots can use MR to place cartoon annotations on themselves or on their surroundings. While it is possible for robots to use other display techniques to render cartoon art, we chose MR as it is very flexible in its ability to augment interaction spaces: it can superimpose information anywhere on the robot's body, place elements in the immediate vicinity of the robot (e.g., over its head), or also allow robots to leave virtual elements behind in the environment.

In this paper, we present and explore the idea of robot expressionism, and in particular the use of cartoon-art. We start by discussing how our work relates and contributes to to the current state-of-the-art. Following, we present the idea of robot expressionism in detail, and explore the use of cartoon-art by robots. Finally, we present a test-bed implementation that allows for diverse and rapid prototyping of cartoon-art interfaces, including scenario implementations and informal pilot study results.

\section{RELATED WORK}

In this section we discuss the issue of believability and acceptance of social robots by human users, offer a review of robotic platforms related to our work, the existing use of cartoon art for communication, and how MR has been used with robots. We present this section to outline the context of our work and our contributions.

\subsection{The Uncanny Valley}

The uncanny valley is a theory that discusses the relationship between a robot's visual resemblance to a human and our perception of the robot [16]. As a robot becomes more human-like, there is a point when the robot appears human enough for us to believe on some level that it is human, only to (sometimes rudely) discover that it is not. At this point, such a robot is said to be perceived as uncanny and is not well accepted [16]. This dropped region of believability is called the uncanny valley, and two solutions to the problem are provided: build robots that convince us they are human (such as androids), thus emerging from the uncanny valley, or design stylised robots which do not appear human, avoiding the problem alltogether.

Some claim that the uncanny valley derives from a more complex relationship which separates the human likeness into two independent categories: behaviour and appearance. Likeness to human appearance can put a robot into the uncanny valley while likeness in behaviour will not. However, given a robot which falls into the uncanny valley, increasing the likeness to human behaviour can help pull it out of the valley without other change to appearance $[11,14]$.

\subsection{Robotic Platforms}

The general goal of androids is to be nearly indistinguishable from humans in everyday situations. While not yet believably human, Osaka University's Repliee Q1 is arguably one of the most realistic androids ever constructed to date. She not only incorporates a human-like physical appearance, but research into eye contact, gaze, gestures, physical reactions and language use make her behaviourally human-like. [11, $12,14,15]$. A similar approach has been taken by Leonardo, a robotic project by the MIT Media Lab [8]. Leonardo does not look human, but is able to interact with people using human-like gestures and facial expressions, making him behaviourly, but not visually, similar to humans. Leonardo has been successful in achieving intuitive interaction by combining these abilities simultaneously, resulting in the impression of an intelligent animal rather than a human-like robot [8].

The Robovie series of robots were explicitly designed for social-level communication with the general public [10, 19]. They have a mechanical appearance and use speech, movement, and gestures for interaction. These robots were used as platforms for a study involving science museum guide robots: four robots were placed in a museum for more than two months and interacted with tens of thousands of visitors [19]. Most of these visitors expressed that they had a good experience and little or no anxiety about the robots, but the main complaint was that the robot capabilities were too limited to allow for in-depth interaction [19].

The Roomba is a commercially successful vacuum robot series from iRobot [9] and is unique in that it is both affordable and has effective utility. One of the challenges faced by the roomba is that it must fit into home environments and be sensitive to existing in-home cultures and routines where it must co-exist with families. A recent study considered this, and explored how the Roomba would fit into and affect the social structure of families [6]. The results suggest that in addition to being functionally useful, the Roomba became a part of the home and in a sense, part of the family. In many cases, people anthropomorphised the roomba and gave it emotions and character, as well as a name, and some admitted talking to it while it worked. 


\subsection{Cartoon Art}

A cartoon, traditionally, is a preperatory work or rough sketch of a completed piece of art [3]. In modern times, however, the word cartoon has come to represent the stylistic art often found in comic strips and animated cinema. Scott McCloud's book Understanding Comics: The Invisible Art [13] explores the language of comics and cartoon art, and argues for the recognition of these as a respected artform. This book discusses the communication language and symbols used in comics, and provides a base understanding of many techniques used by comic artists.

Artistic techniques found in cartoon art, such as exaggeration and simplification, have been used throughout human history as far back as petroglyphs and cave writings. These techniques have found their way into computers, as cartoon art has flourished in interfaces including icons and cursors, video games, and wizards including the famous Microsoft Clippy assistant.

\subsection{Mixed Reality Robotic Interfaces}

There has been little work to date on using MR for HRI. Existing efforts have been primarily concerned with direct robot control or tele-presence, such as a project by Giesler et al. that uses MR to allow users to dynamically create a route map for the robot using a wand and MR equipment [7]. The system works directly within the robot's physical working space and augments the user's vision with a MR map in real-time [7]. However, this effort focuses on the use of MR for direct control of the robot rather than social interaction.

Dragone et al. uses MR to add avatars to robots in an attempt to gain transferability between HCI and HRI. The project places a virtual character at the top of a robot, providing an expressive interface that is easily adapted and changed [4]. By using avatars, robots can communicate using human-like characteristics: they use simple animations, facial expressions and gestures. For example, an agent may nod or salute to acknowledge a command, or shrug to indicate a lack of understanding.

\subsection{Robot Expressionism through Cartooning}

We believe that cartoon art will help robots to avoid the uncanny valley as it uses stylistic, rather than realistic, representations, and increases a robot's behavioural likeness to humans. In effect, we are increasing behavioural likeness without increasing visual likeness. This approach is similar to Leonardo and the Robovies which use human communication without trying to appear human. However, cartoon art is different in that it allows a robot to break free from the limitations of its physical body and gesture capabilities, freely adding colour, animation, and annotations at any location on or around its body. Robots can also express cartoon art at a distance from their body, for example, mobile robots could leave behind cartoon elements at physical locations as they move around. Cartoon art does not replace, but rather can compliment gestures and body language expressions for rich interaction.

We argue that cartoon art is simple in nature and avoids increasing users' expectations of intelligence and complexity in robots. Robots that use more complex methods like speech (such as the Robovie) are currently limited to few and specific conversation or interaction templates due to the complexity of the technique involved. As shown with the Robovies, users can have high expectations of robots that use such complex methods to communicate with them, and then are sometimes surprised and disappointed by the lack of actual depth of interaction. While cartoon art is not a replacement for speech and other metaphors, its simplicity helps it to avoid these complexity problems.

Results from the Leonardo project and the Robovie museum study suggest that robots that make convincing use of human communication techniques will be fairly well accepted by the general public. Robots that use cartoon art are expected to have similar results, as techniques from cartoon art are widely understood and accepted.

While the Roomba keeps a mostly mechanical image, using buttons, simple blinking lights and beeps for interaction, users still tend to anthropomorphise it. Cartoon art will offer a simple but powerful tool that this class of robots can use for social interaction, capitalising on the users' tendency to anthropomorphise, and requiring a minimal amount of additional hardware. While our implementation uses advanced MR systems, robots could use simplified displays or pre-designed lights and shapes to convey cartoon art. We use MR as a flexible medium that allows us to explore the various ways in which cartoon art can be used by robots, but it is not necessarily the most effective or simplest way for cartoon art to be realised.

Dragone et al.'s robotic avatars are meant to be the focus of interaction; the physical robot is simply a mechanical means for mobility and interaction with the environment, and the user is expected to ignore most of the physical attributes of the robot and focus on the avatar. Instead, we point out that cartoon art techniques can augment and compliment existing robotic interaction metaphors such as speech and gestures, and can capitalise on and work with the physical nature of the robot. For example, robots such as Leonardo could use cartoon art to expand on their communication ability while still using their expressive physical gestures.

\section{ROBOT EXPRESSIONISM}

The term expressionism refers to a movement in the arts during the early part of the 20th century that emphasised subjective expression of the artist's inner experiences and state of mind [5]. The expressionism movement was part of a shift in western art where artists were decreasingly bound by the restraints of direct representation and were allowed much more freedom in their expressive styles, giving a more subjective representation of what they wanted to convey.

With the digital nature of robots, direct representation is trivial; a robot can always list or display the trillions of bits describing its condition and state. However, similar to the human artists during the expressionism movement, we suggest the term robot expressionism to indicate that robots can provide a layer of insight into their internal state and intentions above and beyond simply offering raw data or direct representation. This includes a conscious recognition of the fact that these high-level interfaces are artistic, and can not always be treated in a strictly technical fashion. 
Using the term robot expressionism encourages interface designers to see robotic interaction as rich, unique, and emphasises the subjective and artistic nature of high-level interaction. Rather than striving for purely functional interfaces with low-level communication, robot expressionism considers the effects, both negative and positive, of the resulting abstraction. The effect is similar to the difference between technical writing and literature; while technical writing (and low-level robotic interfaces) focuses on clear and concise representation, literature (and robot expressionism) adds artistic depth and richness to the work.

While some expressionism techniques, such as shrugging in the example above, are culture specific, this is not necessarily always the case; there are techniques with stronger, or less strong, cultural dependence. For example, an entertainment robot may use speech and slang specific to a particular small geographical region, while many facial expressions employed by androids may span many, if not all, cultures.

In general, robot expressionism implies an abstraction of a robot's digital condition for the purpose of providing contextual insight into the robot's state. Robot expressionism techniques also employ artistic elements and have a level of ambiguity that increases with the level of abstraction and artistic content. This ambiguity, however, can be desireable as it is often accompanied by interaction richness and depth. For example, an expressionist robot may shrug if its artificial intelligence systems could not solve a question. While this is ambiguous as it offers no insight into the reason for the lack of knowledge, the shrug is a rich gesture that adds character to the robot and provides high-level insight into the situation: the robot simply did not know the answer. This approach to communication may be more intuitive to humans and interesting as high-level interaction, particularly when dealing with the general public.

Social robots, in general, are using robot expressionism. These robots abstract away from their algorithms and digital states, offering humans information using less direct gestures, speech, and other human-communication metaphors.

\section{CARTOON ART FOR ROBOT EXPRESSIONISM}

We propose to teach robots a form of expressionism by instructing them to interact using techniques from cartoon art. These robots will communicate using an anthropomorphic view of what they would expect a human to understand as their subjective state. Cartoon-art abstraction helps to strip away superfluous details of direct representation and focuses on the defining features which are easily understood. For example, a robot which is faced with a situation that it does not understand may portray itself as a confused creature using a stylised and simplified facial expression and a question mark above it.

In particular, we are interested in how robots can use the icons, varying text styles, and simplified and exaggerated facial expressions and gestures commonly found in cartoon art. We refer to icons in cartoon art as annotations such as movement lines, dust marks, and dizzy stars or heart symbols. These give robots a method to show movement, emphasis, or emotion such as happiness or surprise. Second, varying text is used in comics and cartoons for emphasis and variance in emotion or intent, both in dialogue and as added situational information. Text may be varied from typeset to hand printed or handwritten text, and can have varying colours, fonts, weight, decorations such as patterned letters or stylised letters. A robot can use these variations to add subtle or obvious overtones and meaning to text and letters. In addition, text is often combined with thought or speech bubbles, both of which can also be stylised in their own way. Third, cartoon and comic art often use simplified and exaggerated facial expressions and gestures as a way of clearly conveying an emotion or state, often anthropomorphising otherwise inanimate objects. These expressions usually focus on the expressive parts of the face, using eyes, wrinkle lines, eyebrows, and mouth, among other things, to convey a human-like emotion or state. The exaggerated nature allows for much more emphasis than in real life, such as oversized eyes or mouth, and very clear emotional state communication. Robots can use these facial-expression techniques to anthropomorphise itself or other objects, clearly communicating a human-like emotion. Finally, robots can simultaneously use a combination of icons, text, and facial expressions. For example, a face may have heart-shaped or swirly eyes, or text may be decorated with various symbols.

Techniques from cartoon art can be used by robots in three primary ways. It can be used to: augment the body of the robot directly (for example, add a face to a robot), augment the immediate area around the robot (for example, place icons above a robot's head), or augment anywhere within the environment (for example, a robot can annotate other physical objects). This idea is based loosely on our early research into the use of MR for HRI [20].

A robot can augment its own body with cartoon art to add and increase the expression of any existing physical communication. For example, a robot may give itself an exaggerated face or iconic tattoos of motion lines or dirt. Following, it can place items around its body such as motion lines, rotating stars showing a state of dizziness, or expressive words such as 'VROOOM' to give the impression of speed. It can also use thought or speech bubbles with a combination of text or icons to give a more explicit form of communication; we call these Bubblegrams in [20]. The ability to leave cartoon-art elements in the environment allows for many possibilities, as a robot can anthropomorphise or give focus to any object, such as using icons representing sound to exaggerate the noise from a stereo it deems to be too loud. In addition, a robot can leave elements in an environment, elements that persist even after the robot is no longer there; we call these Thought Crumbs in [20], named after Hansel and Gretel's bread crumbs. Thought Crumbs can be materialise as a variety of cartoon art entities, for example a message in a thought bubble left for a human, or cartoon-like muddy footprints showing where a robot has been.

\section{JEEVES: CARTOON ART TEST BED}

As a means of exploring and evaluating the use of cartoon art for robot expressionism, we developed a test-bed system called Jeeves. This system contains an autonomous robot, uses MR for visualisation, and uses computer vision for locating the robot positioning. MR allows us to place full 


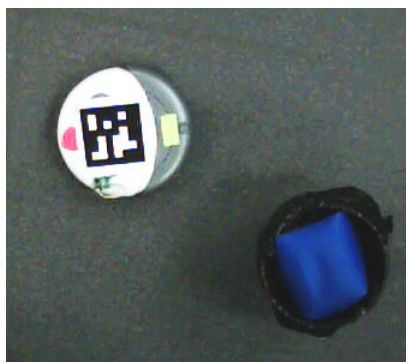

(a) Original image

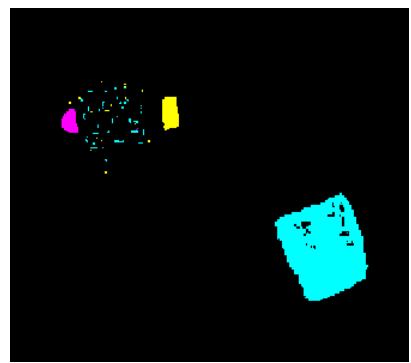

(b) Colour segmented image.
Figure 2: Tracking Jeeves using colour segmentation. [2]

colour, dynamic and animated annotations anywhere on the robot or in the environment.

The goal of this system is to provide a testing environment where tasks, implementations and scenarios can be quickly developed and prototyped. It should also allow for easy integration of various robots, environments, MR medium (HMD, PDA, etc), and of course, a full range of cartoon art rendering possibilities. We will not focus on input to the system and instead we focus on the ways in which robots can integrate and use cartoon art as part of their communication vocabulary.

There are three main components to our system: the robot, the control module, and the MR module. In our current implementation, we use a Roomba vacuum robot, a PC and vision system as the control module, and another PC, vision, and display system for the MR.

\section{JEEVES IMPLEMENTATION}

The Roomba robot is a very simple device and is not directly programmable, but has a serial port that can be used for direct control. We installed a bluetooth module [18] that connects to the Roomba's serial port and communicates with the controlling module. This gives the controlling module full drive and light control, and gives access to the Roomba's sensors, including the bump sensors and top buttons.

The controlling module is a stand-alone PC that communicates with the Roomba using bluetooth and passes relevant information to the MR module using TCP. This PC has a web camera situated above the interaction environment providing an overview of the interaction space from above. Images from this camera are processed by computer vision where the location and direction of the robot as well as the location of other objects are extracted. Currently, we are using colour segmentation techniques (see Figure 2 [2]) and are marking the robot and objects with solid colours like yellow or pink.

The MR module is currently a tablet PC that communicates with the controlling module over the wireless network, using the information to decide which annotations to display. To place the annotations, we using both colour segmentation and a development library called AR Tooklit [1] which, with the use of in-scene markers, gives 3D position and orientation. This allows for cartoon-art annotations to be placed properly scaled and oriented in the $3 \mathrm{D}$ scene. The tablet $\mathrm{PC}$ is also equipped with a webcam, such that a user can hold it up and look through the display into the room. The display is augmented, making the tablet a MR device. We also have an HMD equipped with a webcam that allows for video see-through into the scene; this HMD can be simply plugged into the tablet and immediately used to view the Jeeves MR environment.

\section{EXAMPLES AND SCENARIOS}

We have developed scenarios utilising cartoon art that serve as proof-of-concept, and helps show the possibilities and versatility of this approach. We present three examples here: trash Jeeves, the recycle police, and clean-tracks.

Trash Jeeves is a simple scenario where our butler Roomba being obstructed by a garbage can while cleaning the floor. Noticing a near-by user, the butler makes efforts using its physical presence and cartoon art to get the user to assist it: it physically bumps the garbage can and tries to push it while expressing fatigue and annoyance using cartoon art. The cartoon art annotations both augment the robot directly and the vicinity directly around the robot, and utilises simplified facial expressions and cartoon-art icons. We have this scenario fully implemented and functional, and it is used for evaluation in Section 8 (see Figure 3(a)).

The recycle police is an environmentally-friendly Roomba which roams a room environment looking for recycleables. Once found, the Roomba tags the items and continues searching, with the hopes that a human user will notice the tag and recycle the item. This scenario augments the Roomba officer, the direct vicinity and leaves MR thought crumbs in the environment, and uses cartoon-art facial expressions, icons, and text to communicate with human users. Screen shots of actual content are shown in Figure 3(b).

Clean-tracks is a cartoon-art addition to the regular Roomba which leaves tire tracks behind on the floor to show where it has cleaned. These tracks persist until the roomba is done cleaning the room; this is useful as a human user can get a sense of progress of the Roomba's work and can know which areas have been cleaned. This scenario uses thought crumbs to place tire tracks behind the Roomba as it moves.

\section{PRELIMINARY EVALUATION}

We performed a preliminary evaluation of our system focusing on the trash roomba scenario. For this, we asked participants to use our test bed in a basic interaction scenario and to comment about the experience using a Think Aloud evaluation technique. At this point, we were interested in the users' initial reactions to the hardware system, the use of cartoon art, and the scenario. Four users, all graduate students in our lab who were not familiar with our work, participated and gave feedback.

All users enjoyed the interface, saying that it was "cute", "fun", and "interesting". All participants also anthropomorphised the Roomba, and one user mentioned at one point that the Roomba seemed 'really angry'. While everyone mentioned that they noticed the roomba was stuck, only one user attempted to do anything as they moved the trash can out of the robot's way (see Figure 3(a)). Finally, one 

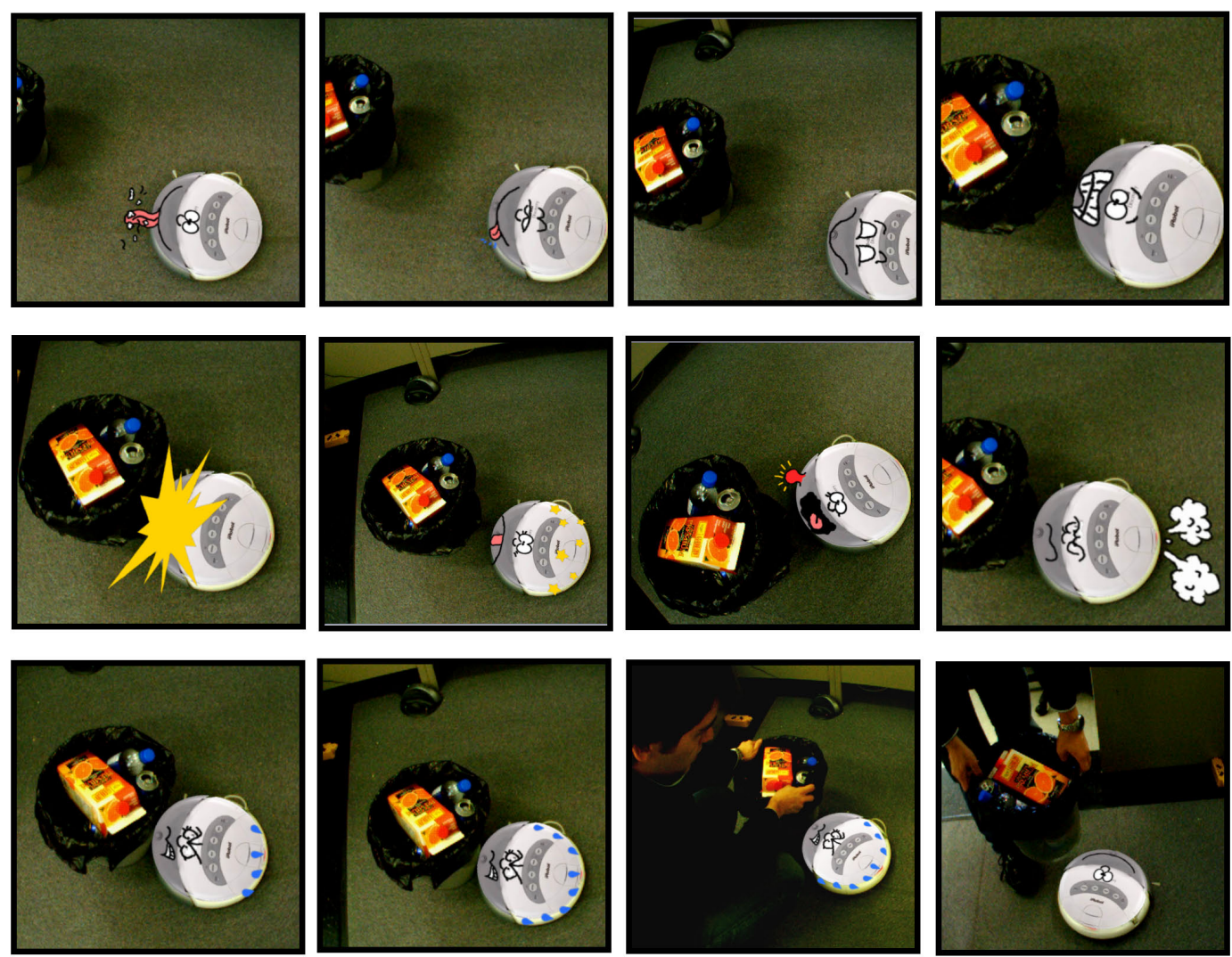

(a) Hard at work, the cleaning Roomba needs assistance to move a trash can.
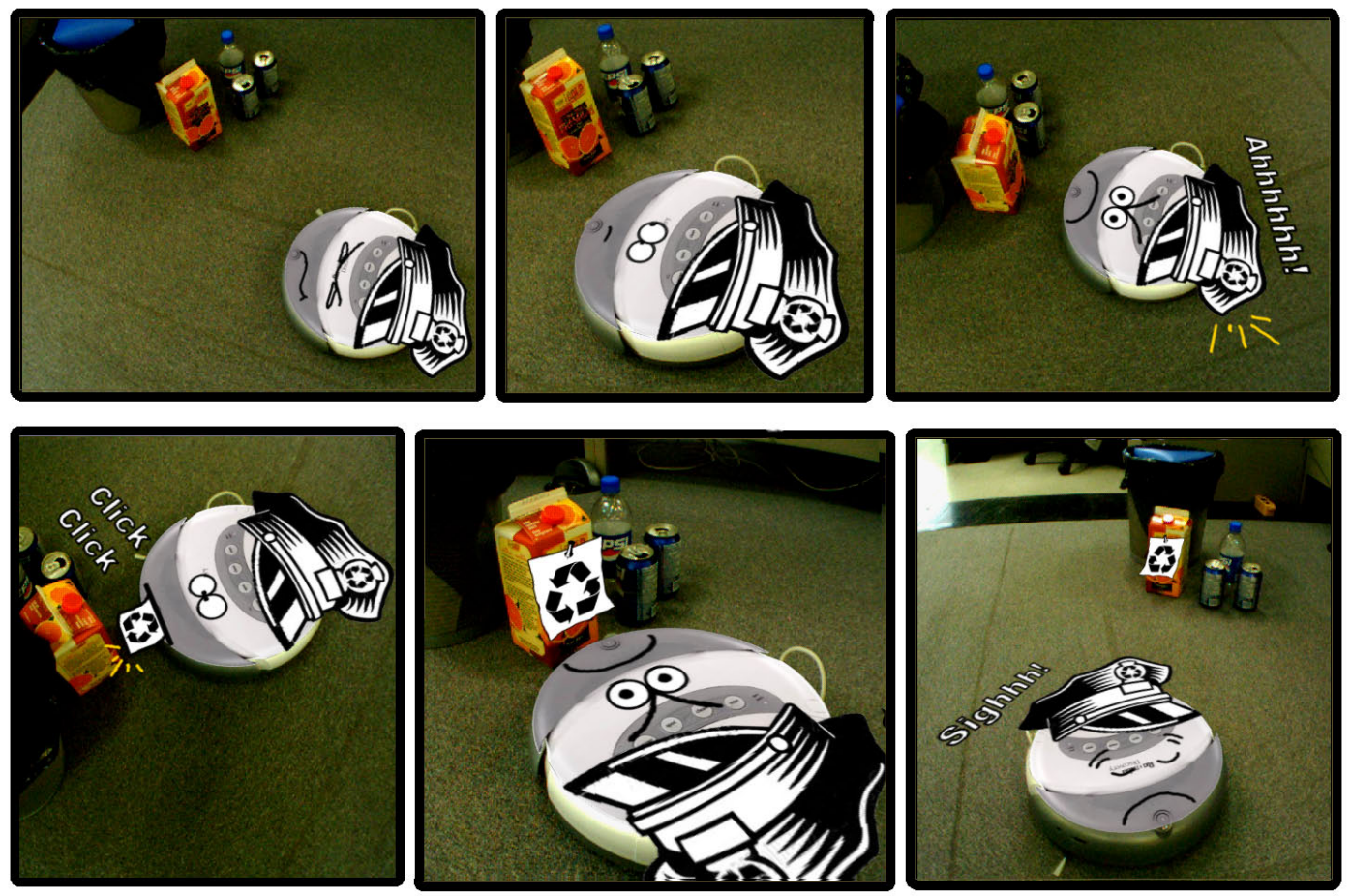

(b) The recycle police relentlessly tickets any recycleable item.

Figure 3: Screenshots of our Jeeves prototype: Trash Jeeves(top) and the recycle police(bottom) 
user mentioned that the augmented Roomba seemed "more personal than most electronics", and that this is an improvement over their existing home appliances.

The users also had some suggestions about the work. One user voiced concern that the cartoon art may be flashy and annoying, and that a robot should be careful as to how it uses these techniques. Another participant complained about the tablet MR interface, stating that they put more energy into working with the MR interface than viewing the cartoon art. Finally, one user complained about the quality of the animation and that the interaction may be improved with the addition of sound.

This preliminary evaluation demonstrates some of the potential of our approach: all users naturally understood the Roomba's state, they all anthropomorphised the roomba, and at least one user viewed the Roomba on a more social level as a result of the cartoon art. However, participants also raised some important concerns about our system and the potential caveats of our approach: when developing cartoon art content, we have to be careful to fit the level and types of cartoon art to the task and scenario at hand. Too subtle or too distracting art may not only be ineffective but can be frustrating for users. Also, we have to be careful to make the MR system as usable and seamless as possible, to avoid MR technical limitations from interfering with the cartoon art. Finally, when creating cartoon art the visual quality of the content is important, including the quality of the art and the animation; problems in the content can disrupt the communication with the user.

\section{FUTURE WORK}

First, there is a need for a theoretical foundation, including a framework for understanding the intersection of expressionism, cartoon art, and MR. This also includes a taxonomy for classifying the use of cartoon-art robot expressionism, a

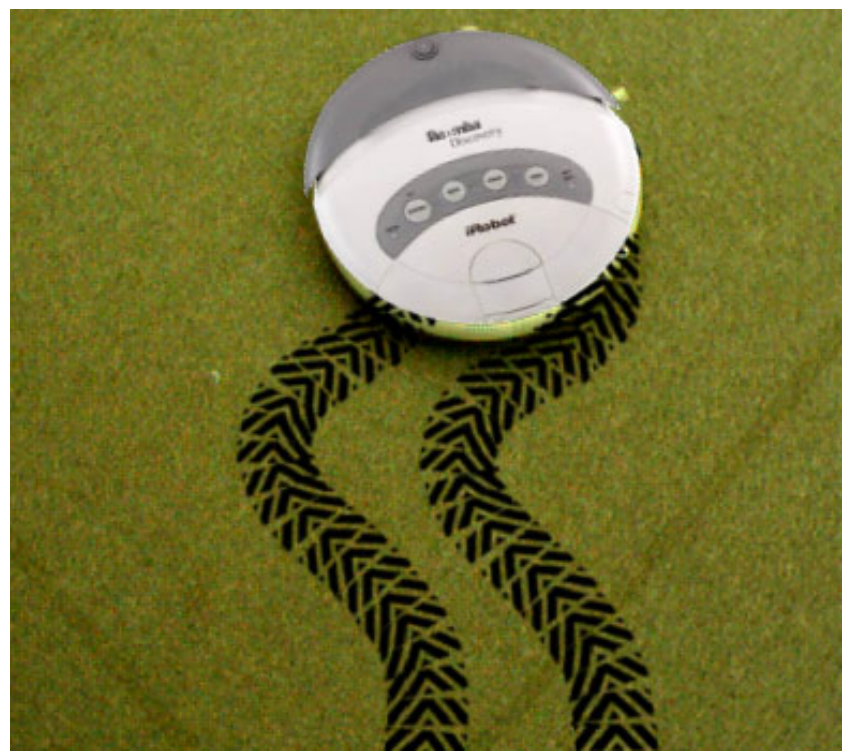

Figure 4: Screenshot of clean-tracks: Jeeves leaving tire tracks behind in the environment. cartoon-art vocabulary for building HRI content, and a set of heuristics for design.

Secondly, we plan to expand our test bed and implement a full set of MR-based display techniques, including vision and various displays from HMDs to projectors and screens.

Thirdly, we want to develop complete, detailed HRI interfaces dedicated to specific and valid robot tasks using Jeeves. Selected tasks will utilise the robotic-expressionism based MR cartooning and would possibly take place in lab-settings using available off-the-shelf robots.

Finally, we need to thoroughly study and evaluate our interfaces new interaction paradigm, techniques and interfaces, including an analysis of their effectiveness and ability to improve the quality of interaction relative to existing, more orthodox, robotic interfaces.

\section{CONCLUSION}

Communication between humans relies on a large amount of common ground and various levels of inter-personal understanding. Because of this, natural communication between humans and robots is often a challenge; robots are digital entities while humans are not. We suggest to approach this problem by teaching robots cartoon-art expressionism, abstracting bits and bytes into higher level cartoon annotations and allowing robots to offer insight into their state using the well-understood language of cartoon and comic art. We believe that this approach can provide robots with powerful expressive ability understood by the general public.

We believe that our cartoon art approach is an effective medium for robotic interfaces for various reasons: It avoids the uncanny valley by increasing behavioural likeness to humans without attempting to increase visual likeness to humans, and it allows robots to break free from many existing physical limitations in expression by allowing them to display dynamic cartoon art on their body, around their body, or anywhere in the environment. In addition, cartoon art is a rather simple medium, using basic shapes and colours, and a clear language understood across cultures and languages. Finally, cartoon art can be used in conjunction with existing robotic interfaces, strengthening and augmenting the other approaches.

As a means of realising our ideas, we provide a test bed and implementation called Jeeves, utilising MR technology and a Roomba robot, allowing for rapid and versatile prototyping of cartoon-art interfaces and scenarios. Using this test bed, we conducted a preliminary user study to get initial feedback on our approach; these results were promising, suggesting there is merit to continuing our work, and offering insight into its limitations and risks.

In conclusion, we see robot expressionism through cartooning to be an effective, valid, and interesting approach to human-robot interaction, one that can provide a simple and intuitive interface for users.

\section{REFERENCES}

[1] M. Billinghurst, H. Kato, and I. Poupyrev. The MagicBook: moving seamlessly between reality and 
virtuality. IEEE Computer Graphics and Applications, 21(3):6-8, May/June 2001.

[2] J. Bruce, T. Balch, and M. Veloso. Fast and inexpensive color image segmentation for interactive robots. In Proc. IEEE/RSJ IROS '00, volume 3, pages 2061-2066, Los Alamitos, CA, Oct. 2000. IEEE Press.

[3] cartoon. (n. d.). The American Heritage ${ }^{\circledR}$ Dictionary, $4^{\text {th }}$ Edition. WWW, http://www.thefreedictionary.com/cartoon, Visited May 17, 2006, 2003.

[4] M. Dragone, T. Holz, and G. M. O'Hare. Mixing robotic realities. In Proc. IUI '05, pages 261-263, New York, NY, 2006. ACM, ACM Press.

[5] expressionism. (n.). The American Heritage ${ }^{\circledR}$ Dictionary, $4^{\text {th }}$ Edition. WWW, http://www.thefreedictionary.com/expressionism, Visited Jun 8, 2006, 2003.

[6] J. Forlizzi and C. DiSalvo. Service robots in the domestic environment: a study of the roomba vacuum in the home. In Proc. HRI '06, pages 258-256, New York, NY, 2006. ACM, ACM Press.

[7] B. Giesler, T. Salb, P. Steinhaus, and R. Dillmann. Using augmented reality to interact with an autonomous mobile platform. In Proc. ICRA '04, pages 1009-1014, Los Alamitos, CA, 2004. IEEE Computer Society, IEEE Press.

[8] M. M. Group. Leonardo. WWW, http://robotic. media.mit.edu/projects/Leonardo/Leo-intro.html, Visited May 22nd, 2006, 2006.

[9] iRobot. Roomba. WWW, http://www.irobot.com/consumer, Visited May 22nd, 2006, 2006 .

[10] H. Ishiguro, T. Ono, and M. Imai. Robovie: an interactive humanoid robot. Industrial Robot: An International Journal, 28(6):498-503, Oct. 2001.

[11] K. F. MacDorman, T. Minato, M. Shimada, S. Itakura, S. Cowley, and H. Ishiguro. Assessing human likeness by eye contact in an android testbed. In Proc. CogSci '05. Cognitive Science Society, Inc., 2005.

[12] D. Matsui, T. Minato, K. F. MacDorman, and H. Ishiguro. Generating natural motion in an android by mapping human motion. In Proc. IEEE/RSJ IROS '04, pages 1089-1096. IEEE Computer Society, 2005.

[13] S. McCloud. Understanding comics-the invisible art. HarperPerennial, New York, NY, USA, First HarperPerennial edition, 1994.

[14] T. Minato, M. Shimada, H. Ishiguro, and S. Itakura. Development of an android for studying human-robot interaction. In Proc. IEA/AIE '04, pages 424-434, New York, NY, 2004. ACM, ACM Press.

[15] T. Minato, M. Shimada, S. Itakura, K. Lee, and H. Ishiguro. Does gaze reveal the human likeness of an android? In Proc. IDCL '05, pages 106-111, Los Alamitos, CA, 2005. IEEE Computer Society.

[16] M. Mori. Bukimi no tani: The uncanny valley (in japanese). Energy, 7:33-35, 1970. Translation at CogSci '05 workshop: toward social mechanisms of android science. WWW,

http://www . androidscience.com/theuncannyvalley/ proceedings2005/uncannyvalley.html.

[17] D. Norman. Emotional design: why we love (or hate) everyday things. Basic Books, New York, USA, 2004.

[18] RoombaDevTools. Rootooth. WWW, http://www.roombadevtools.com, Visited Aug 30th, 2006,2006 .

[19] M. Shiomi, T. Kanda, H. Ishiguro, and N. Hagita. Interactive humanoid robots for a science museum. In Proc. HRI '06, pages 305-312, New York, NY, 2006. ACM, ACM Press.

[20] J. E. Young and E. Sharlin. Sharing spaces with robots: an integrated environment for human-robot interaction. In Proc. ISIE '06, pages 103-110, Cambridge, UK, Apr. 2006. Microsoft Research. 